\title{
Inflammation and perforation of a solitary diverticulum of the cecum. A report of 5 cases and literature review
}

\author{
J. Ruiz-Tovar, M. E. Reguero-Callejas ${ }^{1}$ and F. González Palacios ${ }^{1}$ \\ Departments of Surgery and 'Pathology. University Hospital Ramón y Cajal. Madrid, Spain
}

\begin{abstract}
Solitary diverticulum of the cecum is a benign condition uncommon in the Western world, and with a higher incidence in Asian population. They are usually asymptomatic, and manifest clinically only with complications such as inflammation, perforation, or bleeding. They are a rare cause of acute abdominal pain, clinically similar to acute appendicitis, with tenderness in the right lower quadrant, fever, and leukocytosis. In spite of the information provided by ultrasonography or CT scans, a correct preoperative diagnosis is still difficult to reach, and is usually arrived at in the operating theater; differentiation from a neoplasm may be also sometimes complicated, and a wide surgical resection is usually required for such cases.

We report on 5 cases of inflammation and perforation of a solitary cecal diverticulum, and perform a literature review.
\end{abstract}

Key words: Solitary diverticulum of the cecum. Right diverticulitis. Congenital diverticulum of the cecum. Right iliac fossa. Right lower quadrant pain.

Ruiz-Tovar J, Reguero-Callejas ME. Inflammation and perforation of a solitary diverticulum of the cecum. A report of 5 cases and literature review. Rev Esp Enferm Dig 2006; 98: 875880 .

\section{INTRODUCTION}

Solitary diverticulum of the cecum is a benign condition that was first described by Potier in 1912 (1). It is uncommon in the Western world, but is highly prevalent in Asiatic populations (2). In the West, most diverticula of

Recibido: 13-06-06.

Aceptado: 13-07-06.

Correspondencia: Jaime Ruiz-Tovar. Corazón de María, 64, 7º J. 28002 Madrid. e-mail: jruiztovar@gmail.com the cecum are solitary (3). Solitary diverticulum of the cecum is thought to be a congenital lesion arising as a sacular projection during the sixth week of embrionary development (4). It is considered a true diverticulum having all the layers in the colonic wall, including the muscularis mucosae and muscular layer, and is located next to the ileocecal valve $(5,6)$. They are usually asymptomatic, and only manifest when complicated with inflammation, perforation, or bleeding. They are an unfrequent cause of acute abdominal pain, but manifestations are clinically identical to acute appendicitis -pain in the right lower quadrant, fever, and leukocytosis. A correct preoperative diagnosis is difficult to reach, and the condition is usually identified during surgery (2).

\section{CASE 1}

A 24-year-old woman presented in the Emergency Department with pain in the right lower quadrant for the last 3 days; she had no fever, vomits, or altered intestinal transit. Physical examination revealed the presence of a mass in the right iliac fosse that was painful with no signs of peritonism. Laboratory tests were normal except for a high fibrogen plasma levels. Ultrasonography showed an uncertain hyperechogenic image in the right iliac fosse. An exploring laparotomy was performed, which identified a mass in the cecum. The patient underwent ileocecal resection, and recovered uneventfully. The pathological study revealed a perforation of a solitary diverticulum of the cecum with extensive destruction of the cecal wall.

\section{CASE 2}

A 44-year-old woman is admitted for intermittent abdominal pain with colic characteristics in the right lower quadrant and periumbilical region for 15 hours, accompa- 
nied by nausea and vomiting, but without fever. Physical examination revealed the presence of a mobile mass in the right iliac fosse that was painful with signs of peritonism. Lab tests showed leukocytosis with neutrophilia. A CT scan revealed an image suspicious of intussusception with a tumor within. During laparotomy an intestinal intussusception was seen with a tumor in the cecum -a right hemicolectomy was carried out. The pathological study established the presence of a solitary diverticulum of the cecum with acute diverticulitis and changes consistent with bowel intussusception. The patient recovered satisfactorily.

\section{CASE 3}

An 82-year-old woman presented in the Emergency Room for diffuse abdominal pain for 3 days accompanied by fever of $38{ }^{\circ} \mathrm{C}$, nausea and vomiting, and no diarrhea. Exploration revealed diffuse abdominal tenderness that was increased in the right iliac fosse, with a positive Blumberg's sign. CT scans showed a tumor in the cecum surrounded by an inflammatory plate. An exploring laparotomy was performed, which revealed a tumor in the cecum's posterior wall that included the ovary and right salpinx. The patient underwent a right hemicolectomy and right anexectomy. The pathological study revealed a perforated solitary diverticulum of the cecum with an inflammatory plate including the ovary, with no neoplastic evidence at any level. The postoperative period was uneventful.

\section{CASE 4}

A 30-year-old woman presents at the Emergency Department for abdominal pain in the right lower quadrant for a few hours, with neither fever nor autonomic symptoms. Physical examination revealed peritoneal irritation signs in the right lower quadrant. Ultrasonography showed a mass in the lower cecum. During laparotomy an enlarged posterolateral wall was seen at the cecum, with a normal appendix. An ileocecal resection was performed. The histological diagnosis was a solitary cecal diverticulum with a microscopic perforation.

\section{CASE 5}

A 73-year-old woman with a recent diagnosis of polymyalgia was admitted to the Internal Medicine Department for pain and functional disability in the right leg, fever, and malaise for 3 weeks. Since admission the patient experienced a progressive worsening of her general status with breathing difficulties and mild, painless abdominal distension. All lab tests were normal except for electromyography, which revealed a mixed sensorymotor polyneuropathy. On day 20 after admission the pa- tient experienced a sudden abdominal pain with massive rectal bleeding, which caused her death. A necropsy was requested, which revealed a subacute idiopathic necrotizing myelopathy explaining neurological difficulties, and fecal peritonitis from a perforated solitary diverticulum of the cecum (Fig. 1) as the possible cause of death following a septic/hypovolemic shock.

Other 2 documented cases of solitary diverticulum of the cecum exist in our center, which were incidentally diagnosed when studying the surgical pieces obtained from right hemicolectomy procedures carried out for tumors in the ascending colon and cecum, respectively. The diverticulum had no relation with the tumor, or inflammation signs in any of the two cases.

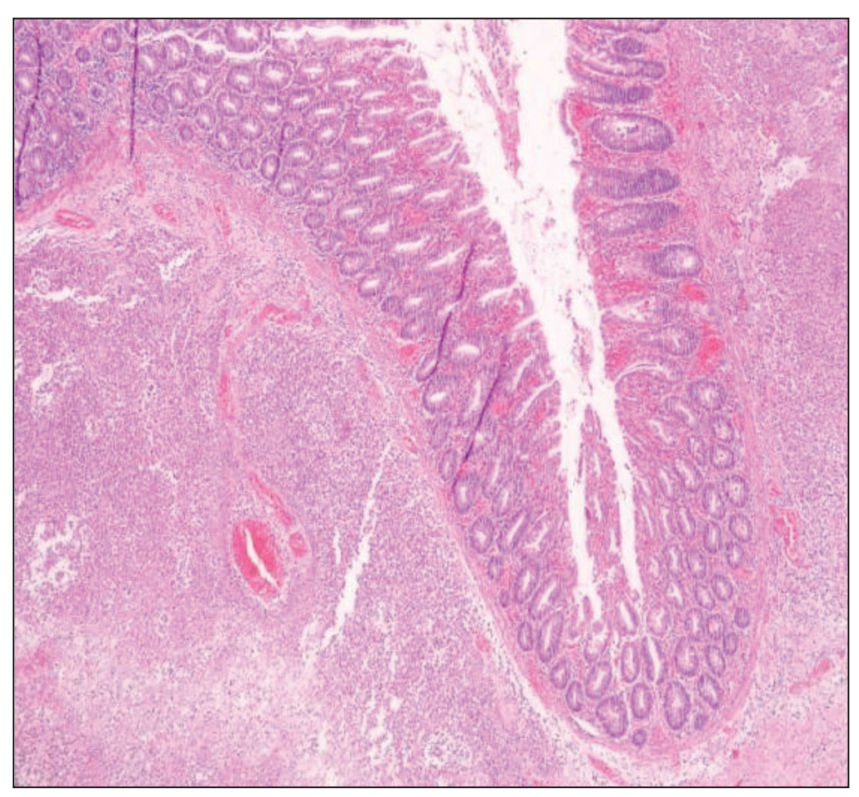

Fig. 1.- HE 20x. A solitary diverticulum of the cecum with acute diverticulitis and fistulization.

HE 20x. Divertículo solitario de ciego con diverticulitis aguda y fistulización.

\section{DISCUSSION}

Most patients with inflammation of a solitary diverticulum of the cecum present with abdominal pain that is indistinguishable from acute appendicitis. The literature advises to suspect this condition especially in young patients of Asian descent presenting with pain in the right lower quadrant of the abdomen for more than $24 \mathrm{~h}$ without nausea, vomiting, or anorexia, or in appendectomized subjects (7). Our experience indicates that suspicion for this condition should extend to all ages, since 2 of our cases were elderly, one was a middle-aged adult, and two were young women. The literature describes no gender predominance, but our 5 cases were women, and the 2 incidentally discovered cases were a man and a woman.

The most frequent clinical manifestations of cecal diverticulitis include abdominal pain in the right lower 
quadrant, fever, and leukocytosis. With these symptoms a differential diagnosis should be established not only with acute appendicitis, but also with other conditions such as gastroenteritis, urinary infection, renal-ureteral colic, pelvic inflammatory disease, or Crohn's disease (8). When a mass in the right lower quadrant is discovered suspicion should not go exclusively towards a neoplasm, but also consider other potential causes such as foreignbody perforation, tuberculosis, actinomycosis, amebiasis, or carcinoid, among others (6).

Abdominal X-rays may reveal a fecalith in up to $50 \%$ of cases, and opaque enema may contribute to diagnosis by defining the diverticulum (6). However, imaging techniques most widely used nowadays for pain in the right lower quadrant include ultrasonography and CT scanning. Ultrasounds have demonstrated a sensitivity of $91.3 \%$, a specificity of $99.8 \%$, and an accuracy of $99.5 \%$ in the diagnosis of cecal diveticulitis, with the diverticulum appearing as a rounded hypoechogenic structure emerging from a colon segment with an enlarged wall (9). CT scans have a sensitivity and specificity of $98 \%$ in the diagnosis of acute appendicitis, and is highly cost-effective; hence some authors suggest its routine use for abdominal pain in the right lower quadrant, which would probably reduce surgeries and hospital stays $(10,11)$. In spite of these data, none of our cases was correctly diagnosed preoperatively, but this can be attributed to the extension of the process at the time of diagnosis in some of them.

The management of non-perforated cecal diverticulitis is controversial. Conservative management consists in wide-spectrum antibiotics if the diverticulum is recognized preoperatively; if it is detected during surgery, management involves appendectomy and postoperative antibiotic therapy. In these cases there is a risk of missing an inflammatory carcinoma; thus, it is considered a valid therapy for Asian populations, where benign right colon conditions are much more frequent than malignancies in this area $(6,8)$. Other authors recommend simple diverticulectomy and appendectomy for cases of non-complicated diverticulitis (6). Fang et al. recommend wide resection, since $29 \%$ of patients undergoing only appendectomy in their study had recurrent episodes of right diverticulitis, with $12.5 \%$ of them requiring a later right hemicolectomy (12).

A number of review works describe that correct intraoperative diagnosis oscillates between 65 and 85\% (13). Even for young patients, if an intraoperative examination does not rule out a malignancy, the correct procedure is to perform a right hemicolectomy with security margins $(2,8,14)$. Other indications for aggressive surgical treatment are the presence of multiple diverticula, and of a great cecal phlegmon. Retrospective studies have demonstrated that right hemicolectomy for cases of right diverticulitis may be carried out safely, with a mortality rate of $1.4 \%(2,15)$.

Chiu et al. highlight the importance of intraperative cecoscopy in the differential diagnosis of diverticulitis with cecal carcinoma; an endoscope is pushed into the appen- dicular stump to visualize the cecal mucosa for lesions suggesting a neoplasm. They conclude that this method makes it possible to exclude the presence of carcinoma on numerous occasions, which would reduce the extension of colonic resection in non-complicated cases (16).

\section{CONCLUSION}

Inflammation of a solitary diverticulum of the cecum is an uncommon occurrence, but should be considered in the differential diagnosis of pain in the right lower quadrant. When no preoperative certainty exists regarding this condition, an exploring laparotomy should be performed; when suspicion of malignancy persists intraoperatively an aggressive surgical resection (right hemicolectomy in most cases) must be performed, as malignant conditions of the cecum are more common than benign disease in Western countries.

\section{REFERENCES}

1. Potier F. Diverticulite and appendicite. Bull Mem Soc Anat Paris 1912; 37: 29-31.

2. Papapolychroniadis C, Kaimakis D, Fotiadis P, Karamanlis E, Stefopoulou M, Kouskouras K, et al. Perforated diverticulum of the caecum. A difficult preoperative diagnosis. Report of 2 cases and review of the literature. Tech Coloproctol 2004; 8: 116-8.

3. Graham SM, Ballantyne GH. Cecal diverticulitis. A review of the American experience. Dis Col Rectum 1987; 30: 821-6.

4. Waugh TR. Appendix vermiformis duplex. Arch Surg 1941; 42: 31120.

5. Sugihara K, Muto T. Diverticular disease of the colon in Japan. A review of 615 cases. Dis Col Rectum 1984; 27: 531-7.

6. Duarte Chedid A, Amaral Domínguez L, Fernández Chedid M, Mello Villwock M, Renato Mondelo A. Diverticulo unico do ceco: experiencia de um hospital geral brasileiro. Arq Gastroenterol 2003; 40: 216-9.

7. Harada RN. Surgical management of cecal diverticulitis. Am J Surg 1993; 166: 666-71.

8. Griffiths EA, Bergin FG, Henry JA, Mudawi AM. Acute inflammation of a congenital cecal diverticulum mimicking appendicitis. Med Sci Monit 2003; 9: 107-9.

9. Chou YH, Chiou HJ, Tiu CM, Chen JD, Hsu CC, Lee CH, et al. Sonography of acute right sided colonic diverticulitis. Am J Surg 2001; 181: 122-7.

10. Fang JF, Chen RJ, Lin BC, Hsu YB, Kao JL, Chen MF. Aggressive resection is indicated for cecal diverticulitis. Am J Surg 2003; 185 : $135-40$.

11. Rao PM, Rhea JT. Effect of computed tomography of the appendix on treatment of patients and use of hospital resources. N Engl J Med 1998; 338: 141-6.

12. Fang JF, Chen RJ, Lin BC, Hsu YB, Kao JL, Kao YC, et al. Aggressive resection is indicated for cecal diverticulitis. Am J Surg 2003; 185: $135-40$.

13. Oudenhoven LF, Koumans RK. Right colonic diverticulitis: US and CT findings. Radiology 1998; 208: 611-8.

14. Keidar S, Pappo I, Shperber Y, Orda R. Cecal diverticulitis: A diagnostic challenge. Dig Surg 2000; 17: 508-12.

15. Lane JS, Sarkar R, Schmit PJ, Chandles CF, Thompson JE Jr. Surgical approach to cecal diverticulitis. J Am Coll Surg 1999; 188: 62934.

16. Chiu PW, Lam CY, Lam SH, WU AH, Kwok SP. On-table cecoscopy: a novel diagnostic method in acute diverticulitis of the right colon. Dis Colon Rectum 2002; 45: 611-4. 